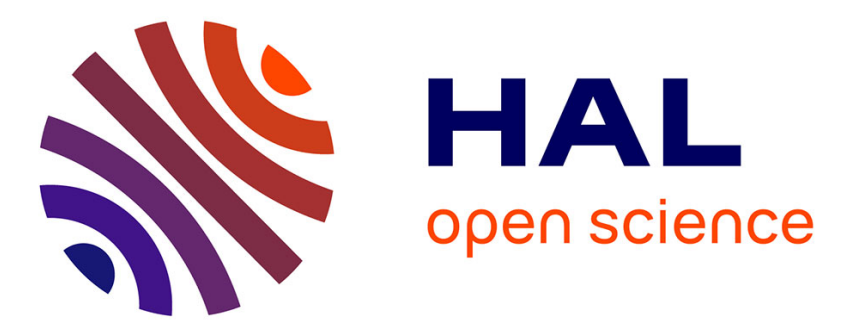

\title{
Lévy processes and stochastic von Bertalanffy models of growth, with application to fish population analysis
}

Tommaso Russo, Paolo Baldi, Antonio Parisi, Giuseppe Magnifico, Stefano

Mariani, Stefano Cataudella

\section{- To cite this version:}

Tommaso Russo, Paolo Baldi, Antonio Parisi, Giuseppe Magnifico, Stefano Mariani, et al.. Lévy processes and stochastic von Bertalanffy models of growth, with application to fish population analysis. Journal of Theoretical Biology, 2009, 258 (4), pp.521. 10.1016/j.jtbi.2009.01.033 . hal-00554572

\section{HAL Id: hal-00554572 \\ https://hal.science/hal-00554572}

Submitted on 11 Jan 2011

HAL is a multi-disciplinary open access archive for the deposit and dissemination of scientific research documents, whether they are published or not. The documents may come from teaching and research institutions in France or abroad, or from public or private research centers.
L'archive ouverte pluridisciplinaire HAL, est destinée au dépôt et à la diffusion de documents scientifiques de niveau recherche, publiés ou non, émanant des établissements d'enseignement et de recherche français ou étrangers, des laboratoires publics ou privés. 


\section{Author's Accepted Manuscript}

Lévy processes and stochastic von Bertalanffy models of growth, with application to fish population analysis

Tommaso Russo, Paolo Baldi, Antonio Parisi, Giuseppe Magnifico, Stefano Mariani, Stefano Cataudella

$\begin{array}{ll}\text { PII: } & \text { S0022-5193(09)00029-0 } \\ \text { DOI: } & \text { doi:10.1016/j.jtbi.2009.01.033 } \\ \text { Reference: } & \text { YJTBI5440 }\end{array}$

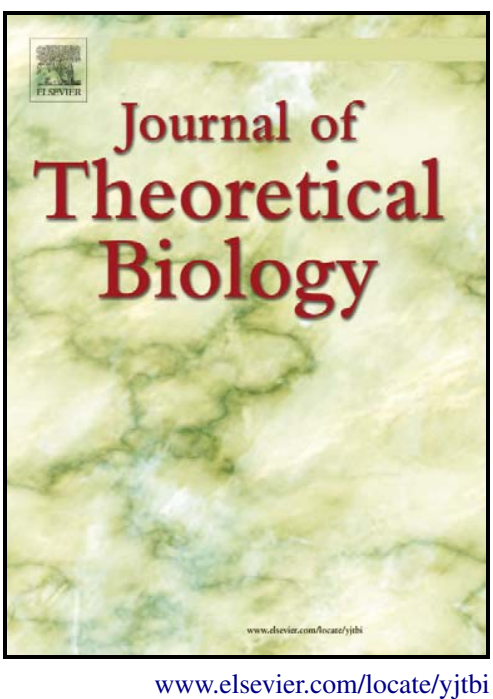

To appear in: $\quad$ Journal of Theoretical Biology

Received date: $\quad 4$ August 2008

Revised date: $\quad 21$ January 2009

Accepted date: 21 January 2009

Cite this article as: Tommaso Russo, Paolo Baldi, Antonio Parisi, Giuseppe Magnifico, Stefano Mariani and Stefano Cataudella, Lévy processes and stochastic von Bertalanffy models of growth, with application to fish population analysis, Journal of Theoretical Biology (2009), doi:10.1016/j.jtbi.2009.01.033

This is a PDF file of an unedited manuscript that has been accepted for publication. As a service to our customers we are providing this early version of the manuscript. The manuscript will undergo copyediting, typesetting, and review of the resulting galley proof before it is published in its final citable form. Please note that during the production process errors may be discovered which could affect the content, and all legal disclaimers that apply to the journal pertain. 


\title{
Lévy processes and stochastic von Bertalanffy models of growth, with application to fish population analysis
}

\author{
Tommaso Russo ${ }^{\text {a,* }}$, Paolo Baldi ${ }^{\text {b }}$, Antonio Parisi ${ }^{\mathrm{c}}$, \\ Giuseppe Magnifico $^{\mathrm{d}}$, Stefano Mariani ${ }^{\mathrm{e}}$, Stefano Cataudella ${ }^{\mathrm{a}}$ \\ ${ }^{a}$ Laboratorio di Ecologia Sperimentale e Acquacoltura - Dipartimento di Biologia - \\ Università di Roma Tor Vergata \\ ${ }^{\mathrm{b}}$ Dipartimento di Matematica - Università di Roma Tor Vergata \\ ${ }^{\mathrm{c}}$ Dipartimento di Scienze Statistiche - Università di Padova \\ ${ }^{\mathrm{d}}$ Consiglio Nazionale delle Ricerche - CNR - Roma \\ ${ }^{\mathrm{e}}$ School of Biology and Environmental Science - University College of Dublin
}

\begin{abstract}
The study of animal growth is a longstanding crucial topic of theoretical biology. In this paper we introduce a new class of stochastic growth models that enjoy two crucial properties: the growth path of an individual is monotonically increasing and the mean length at time $t$ follows the classic von Bertalanffy model. Besides the theoretical development, the models are also tested against a large set of lengthat-age data collected on Atlantic Herring (Clupea harengus): the mean lengths and variances of the cohorts were directly estimated by least squares. The results show that the use of subordinators can lead to models enjoying interesting properties, in particular able to catch some specific features often observed in fish growth data. The use of subordinators seems to allow for an increased fidelity in the description
\end{abstract}


of fish growth, whilst still conforming to the general parameters of the traditional von Bertalanffy equation.

Key words: Individual-based models, Subordinators, Fish growth, Clupea harengus, Fisheries

\section{$1 \quad 1$ Introduction}

2 The modeling of growth and the analysis of intra-population pattern of size

3 variability through time are central topics in animal population biology,

4 since the internal size structure of populations can have a decisive influence

5 on the population dynamics (DeAngelis et al., 1993; Imsland et al., 1998;

6 Uchmanski, 2000; Kendall \& Fox, 2002; Fujiwara et al., 2004). In general,

7 the von Bertalanffy growth function (VBGF - von Bertalanffy, 1957) is the

8 best acknowledged and used relationship to describe the growth of fish and

9 other animals. This equation states that the size of an individual increases in

10 time according to the equation

$$
x_{t}=L_{\infty}\left(1-\mathrm{e}^{-k\left(t+t_{0}\right)}\right)
$$

${ }_{11}$ Where $L_{\infty}$ is the extremal length that is attained as time goes to infinity,

$12-t_{0}$ is the time of conception, at which the size should be 0 and $k$ is a

13 parameter that gives the speed of the process: the larger the value of $k$, the

14 quickest the growth. The VBGF is most commonly used as a descriptive

15 model of size-at-age data (Essington et al., 2001). Nevertheless, equation

16 (1.1) describes the relationship between age and mean length of a

* Corresponding author. Tel.: +390672595974. Fax: +390672595965. E-mail address: Tommaso.Russo@Uniroma2.it 
17 population, whereas the variability among individuals of the same age (e.g.

18 the variance or even the distribution of each cohort) is not included.

19 The popular assumption of gaussianity (Imsland et al., 1998) is clearly a first

20 (rough) approximation in this direction. A natural approach to the problem

${ }_{21}$ of determining the appropriate form of the probability distribution for a

22 population at a given time $t$ is to model first the growth process of the

23 individuals (individual based models, IBM). Nowadays, both in ecological

24 (Arino et al., 2004), evolutionary (Conover \& Munch, 2002; Ernande et al.,

25 2004) and management (Caswell, 2001) contexts, one of the challenges of the

26 researcher is to model how the size of an individual changes in time and

${ }_{27}$ deduce from the growth model which kind of probability distribution models

28 the size of fish at a given age (Lv \& Pitchford, 2007; Fujiwara et al., 2004).

29 A suitable model of growth should account for both individual and

30

31

32

33

34

35

36

37

38

39

40

41

42

environmental variability. In fish, as in other animals, the first source of

variability is rooted in the physiological processes and is the net result of two

opposing processes, catabolism and anabolism (von Bertalanffy, 1938). The

inter-individual variability in growth is the result of several internal (genetic)

and external (environmental) factors which affect these physiological

processes. In fact, while each individual is born with a personal genetic

architecture which primarily determines his growth profile, a number of

physical and biological factors, such as water temperature (Sumpter, 1992),

dissolved oxygen (Brett, 1979), photoperiod (Imsland et al., 2002), and the

availability of appropriate food sources (Rilling \& Houde, 1999), have been

shown to affect growth rates. In order to take into account these aspects as

well as individual variability, a class of individually based model (IBM) was

developed (Lv \& Pitchford, 2007; Sainsbury, 1980; Mulligan \& Leaman, 
43 1992; Wang \& Thomas, 2003; Imsland et al., 2002; Wang, 1999;

44 Gudmundsson, 2005).

45 These models can be classified into two main categories. The first comprises

46 those which consider the inter-individual variability as a stochastic factor to

${ }_{47}$ be added to the general growth curve of the population. The distribution of

48 this factor is the same for all the individuals. In the most recent approaches

49 of this kind (Gudmundsson, 2005; Lv \& Pitchford, 2007; Wang, 1999) some

50 individual-based stochastic models of growth are proposed using a stochastic

${ }_{51}$ differential equations. These models take the general form:

$$
d L_{t}=f\left(L_{t}, t\right)+\alpha\left(L_{t}, t\right) d B(t)
$$

52

Here $L_{t}$ is the size at time $t, f\left(L_{t}, t\right)$ characterizes the deterministic intrinsic growth (drift coefficient) of the individual (the same for all individuals);

$\alpha\left(L_{t}, t\right)$ gives the magnitude of the random fluctuations (diffusion coefficient) and $B(t)$ is a standard Brownian motion, or Wiener process, which is commonly used to model a variety of background and environmental fluctuations in physical, financial and biological contexts, see Karlin \& Taylor (1981) e.g.

The stochastic component of these models is intended to account both for the environment and the inter-individual variability. It should be stressed, however, that (as already remarked in Gudmundsson, 2005) the solution of an equation as (1.2) cannot be monotonically increasing and therefore appears to be unsuitable to model the evolution of the size of an individual. Stochastic models like the one of (1.2) are conversely well suited in order to model quantities, as prices in financial markets, that are characterized by an 

74 Gaussian distribution.

75

oscillating and therefore non monotonic behaviour. This aspect seems to be a drawback for a growth model of several animals like fish. In fact, for fish and other vertebrates, the physiological mechanism of growth in length (i.e. addition of bone material to the axil unit of the skeleton that is the vertebra) leads to a pattern in which the size of an individual is necessarily increasing in time (Weatherley \& Gill, 1987). Let us point out that there exist no Gaussian process which is increasing. Therefore, modeling size variability through an individual growth process, can not give rise to a

Closely related to the models (1.2) are those introduced in Gudmundsson (2005) where it is the derivative of the growth process that is the solution of a stochastic equation. It is possible in this way to obtain a stochastic process that is increasing. These models are interesting and deserve to be tried by testing against real data. Remark however that the mean size at time $t$ of a population following such a model does not follow a VBGF.

The second category of stochastic models suggested so far comprises non-deterministic models in which the individuals of a fish population have different parameters of the VBGF. In this way, each individual has its own triplet $\left(L_{\infty}, t_{0}, k\right)$, that is retained throughout its life (Sainsbury, 1980). Considering the length to age relationship, the length $X_{t}$ at age $t$ of the $i$-th individual with the parameters triple $\left(L_{i}, t_{0, i}, k_{i}\right)$ is given by:

$$
X_{t, i}=L_{i}\left(1-\mathrm{e}^{-k_{i}\left(t+t_{0, i}\right)}\right)
$$

${ }_{87}$ This model displays a monotonic behaviour and considers the inter-individual variability of growth parameters, but does not account for 
the randomness coming from the environment, which can be seen as a

limitation of the model.

Finally, it should be considered that individual growth is a complex energetic

process. Individual length increases only when enough energy from food is available for growth. On the other hand lack of food does not usually lead to a reduction in length, because organisms can lose body mass without shrinking in length (Kooijman, 2000) also because of the presence of a skeleton. Energy may also be allocated to storage for future use, producing "memory" in growth dynamics. Individual organisms encounter and ingest food, which is then assimilated. Assimilated food is transformed into reserve material such as protein and fat. A fixed fraction of the energy from the reserve is used for both metabolic maintenance and growth, and the rest is used for reproduction. Looking at the length of an individual, we suggest that this process determines a pattern in which periods of no growth (determined by scant energy inlet) are separated by periods of growth. If the periods of growth are short, the growth process could be well described by a model allowing for discontinuities, i.e. for instantaneous increases of the length (jumps). This idea is consistent with several observations reported for fish in general and for the species we are going to study (Hinrichsen et al., 2007).

In this paper we introduce two classes of stochastic models of growth that attempt to overcome the drawbacks pointed out above. The main idea developed in this paper is to model the growth process as the solution of a stochastic equation of the form

$$
d X_{t}=\left(L_{\infty}-X_{t-}\right) d Z_{t}
$$


112 where $\left(Z_{t}\right)_{t}$ is a subordinator. These are a class of stochastic processes that 113 are strictly increasing and the solution $\left(X_{t}\right)_{t}$ turns out to be increasing also. ${ }_{114}$ These models enjoy a certain number of desirable features, namely

115 116 randomness;

117 - they are increasing.

118

119

120

121

122 123

124

125

126

127

128

129

130

131

132

133

134 harengus, presented in $\S 5$.

\section{Subordinators}

- $Z_{0}=0$

- the mean size at time $t$ follows a VBGF.

In $\S 2$ we make a quick review on the topics of subordinators upon which our models are built. In $\S 3$ and $\S 4$ some models are developed, attempting to answer to the points exposed above. Finally in $\S 6$ we apply the proposed models to a large set of length-at-age data of Atlantic herring Clupea

A subordinator is a stochastic process $\left(Z_{t}\right)_{t}$ such that

- its paths are right continuous and increasing almost surely;

- has independent and stationary increments.

This means in particular that the distribution of $Z_{t+h}-Z_{t}$ is independent of $t$ for every $h \geq 0$ and that $Z_{t}-Z_{s}$ and $Z_{v}-Z_{u}$ are independent r.v.'s for $u<v \leq s<t$. Also the increments $Z_{t+h}-Z_{t}$ must be stationary, in the sense that their distribution depends on $h$ only and not on $t$. The characterization of such processes (that are particular instances of Lévy processes) has received much attention in time and it is characterized in 
then $M$ is of the form

$$
M_{t}(\theta)=\mathrm{E}\left(\mathrm{e}^{-\theta Z_{t}}\right), \quad \theta \geq 0
$$

$$
M_{t}(\theta)=\mathrm{e}^{t \phi(\theta)}
$$

137

$$
\phi(\theta)=-\theta \gamma+\int_{0}^{+\infty}\left(\mathrm{e}^{-\theta x}-1\right) \nu(d x)
$$

where $\gamma \geq 0$ and $\nu$, the Lévy measure, is a positive measure on $\mathbb{R}^{+}$such that

$$
\int_{0}^{+\infty} \frac{x}{1+x} \nu(d x)<+\infty
$$

Intuitively a subordinator increases as the superposition of a deterministic evolution $t \rightarrow \gamma t$ and of a stochastic process which only makes jumps. These are made at times governed by a Poisson process. More precisely, if $0<a<b$, then $\nu([a, b])$ is the intensity of the Poisson process of the jumps whose size is larger than $a$ and smaller than $b$. This means that if $t$ is the (random) time at which the jump occurs, then the path has, at $t$, a left limit $X_{t-}$ and a right limit $X_{t+}$ that are different, with $X_{t-}<X_{t+}$. For our purposes we shall therefore be much interested in subordinators whose Lévy measure is large to near 0 and gives a small mass only away from 0 . The resulting process would hence be characterized by many very small jumps. 
As every Lévy process, the independence and stationarity properties of the increments implies that if $Z_{t}$ has a finite mean, then

$$
\mathrm{E}\left(Z_{t}\right)=\mu t
$$

for some real number $\mu$ and if it has a finite variance then

$$
\operatorname{Var}\left(Z_{t}\right)=\sigma^{2} t
$$

for some $\sigma^{2} \geq 0$.

It is not possible for a general Lévy measure to compute explicitly the

Laplace transform and/or the density at time $t$ of the associated

subordinator. The next examples introduce some families of subordinators of interest for which these feature can be determined.

- the Gamma process, where the distribution of $Z_{t}$ follows a $\Gamma(\alpha t, \lambda)$

distribution. Recall that such a distribution has a density

$$
f(x)=\frac{\lambda^{\alpha t}}{\Gamma(\alpha t)} x^{\alpha t-1} \mathrm{e}^{-\lambda x}, \quad x>0
$$

and $f(x)=0$ for $x \leq 0$. Of course the increment $Z_{t}-Z_{s}$, having the same distribution as $Z_{t-s}$, has a $\Gamma(\alpha(t-s), \lambda)$ distribution. Its Laplace transform is

$$
M_{t}(\theta)=\left(\frac{\lambda}{\lambda+\theta}\right)^{\alpha t}
$$

so that $\phi(\theta)=-\alpha \log \frac{\lambda}{\lambda+\theta}$. From well-known properties of the $\Gamma(\alpha t, \lambda)$ distribution the statistical indices of interest of a Gamma process are: mean $=\alpha t / \lambda$, variance $=\alpha t / \lambda^{2}$, skewness $=2 / \sqrt{\alpha t}$ and kurtosis $=$ 
$1673+6 /(\alpha t)$.

Here the Lévy measure is

$$
\nu(d y)=\frac{\alpha}{y} \mathrm{e}^{-\lambda y} d y
$$

169 This process therefore gives a high intensity to small jumps (see Fig 1).

170

171

172

(1)

- The inverse Gaussian processes, where $Z_{t}$ is defined as the first time at which a process of the form $\sqrt{\rho} B_{s}+b s$ crosses level $t$. Here $\left(B_{t}\right)_{t}$ denotes a standard Brownian motion and $\rho, b>0$. Its density at time $t$ is, for $x>0$,

$$
f_{t}(x)=\frac{t \mathrm{e}^{b t / \rho}}{(2 \pi \rho)^{1 / 2} x^{3 / 2}} \exp \left(-\frac{b^{2}}{2 \rho} x-\frac{t^{2}}{2 \rho x}\right)
$$

173 It depends on the positive parameters $b, \rho$. Its Laplace transform at time $t$ is

$$
M_{t}(\theta)=\exp \left(-t\left(\sqrt{\frac{b^{2}}{\rho^{2}}+\frac{2 \theta}{\rho}}-\frac{b}{\rho}\right)\right)
$$

174 from which one can derive the expression of the statistical indices of interest:

175 mean $=t / b$, variance $=\rho t / b^{3}$, skewness $=3 \sqrt{\rho /(t b)}$ and kurtosis $=$ $1763+15 \rho /(t b)$.

177 The Lévy measure is

$$
\nu(d y)=\frac{1}{\sqrt{2 \pi \rho y^{3}}} \mathrm{e}^{-\frac{1}{2 \rho} b^{2} y} d y
$$

178 We stress that both the Gamma and the inverse Gaussian process have a

179 Lévy measure that gives much weight to small jumps and that decreases fast 180 at infinity. 
- The $\alpha$-stable process, $0<\alpha<1$ whose Laplace transform is

$$
M_{t}(\theta)=\mathrm{e}^{-c \theta^{\alpha} t} .
$$

For these processes an explicit expression for the density does not exist, unless $\alpha=\frac{1}{2}$. In this case, for $c=\sqrt{2}$, the density is, for $x>0$,

$$
f(x)=\frac{t}{(2 \pi)^{1 / 2} x^{3 / 2}} \mathrm{e}^{-\frac{t^{2}}{2 x}} .
$$

Notice that, as $x \rightarrow+\infty$ the density decreases very slowly and, in particular, both expectation and variance are infinite. Also the Lévy measure decreases very slowly at infinity, therefore allowing very large jumps. We shall see that this model is not well suited for the growth phenomena of interest and we mention it only for reasons of completeness.

To the previous examples one should add the deterministic increasing process: $Z_{t}=\gamma t$. Recall also that the sum of independent subordinators is a subordinator itself.

\section{Modeling by time change}

A first growth model using subordinators can be obtained by time change.

We simply consider that the size of the fish $X_{t}$ at time $t$ is given by

$$
X_{t}=Z_{A_{t}}
$$


195 where $Z$ is the subordinator and $A$ is the von Bertalanffy type function

$$
A_{t}=\left(1-\mathrm{e}^{-k\left(t+t_{0}\right)}\right)
$$

If the subordinator $Z_{t}$ has finite mean, then for some real number $\mu$

$$
\mathrm{E}\left(X_{t}\right)=\mu\left(1-\mathrm{e}^{-k\left(t+t_{0}\right)}\right)
$$

197

and the means follow therefore a VBGF, where $\mu$ plays the role of $L_{\infty}$ (recall

198 that $\left.\mu=\mathrm{E}\left(Z_{1}\right)\right)$.

199 If in particular $Z$ is a Gamma process, then $\mu=\frac{\alpha}{\lambda}$. It is also immediate that, 200 if $Z_{t}$ has finite variance,

$$
\operatorname{Var}\left(X_{t}\right)=\sigma^{2}\left(1-\mathrm{e}^{-k\left(t+t_{0}\right)}\right)
$$

201 for some $\sigma^{2}>0\left(\sigma^{2}=\frac{\alpha}{\lambda^{2}}\right.$ if $Z$ is a Gamma process). Recall that the variance 202 is always increasing in $t$. This fact makes that the models obtained by time 203 change of a subordinator are rather rigid and are in particular unable to 204 account for a non monotonic behaviour of the variance, as is often observed 205 in data. 


\section{Modeling by Doléans exponential}

A more interesting model of growth is given by the process $\left(X_{t}\right)_{t}$ which is obtained as the solution of the stochastic equation

$$
\begin{aligned}
& d X_{t}=\left(L_{\infty}-X_{t-}\right) d Z_{t} \\
& X_{-t_{0}}=0
\end{aligned}
$$

where $\left(Z_{t}\right)_{t}$ is a subordinator. Here $-t_{0}$ denotes the time of conception, at which the size must be considered equal to 0 . If the subordinator $Z$ has a drift $\gamma$ equal to 0 , the solution of (4.10) is a process that remains constant between the jumps of $Z$, whereas it has an increment of size

$$
\left(L_{\infty}-X_{t-}\right) \Delta Z_{t}
$$

whenever $Z$ has a jump of size $\Delta Z_{t}$. The quantity $X_{t-}$ denotes the value of $X$ just before the jump that occurs at time $t$. If we define $Y_{t}=L_{\infty}-X_{t}$, then $Y$ is the solution of

$$
\begin{aligned}
& d Y_{t}=-Y_{t-} d Z_{t} \\
& Y_{-t_{0}}=L_{\infty} .
\end{aligned}
$$

Therefore $Y$ is equal to the Doléans exponential of the Lévy process $-Z$

multiplied by $L_{\infty}$. The Doléans exponential of the Lévy process is a subject

that has received much attention and it is possible to derive an explicit

solution of (4.11), at least if one makes the assumption that the process $Z$

cannot make jumps larger than 1 (which is a quite reasonable assumption, in 
221 our case). Under this assumption the solution of (4.11) is

$$
Y_{t}=L_{\infty} \mathrm{e}^{-\widetilde{Z}_{t+t_{0}}}
$$

${ }_{222}$ where $\widetilde{Z}$ is another subordinator whose Lévy measure $\widetilde{\nu}$ and drift $\widetilde{\gamma}$ are

${ }_{223}$ obtained from $\nu$ and $\gamma$ in an explicit way. More precisely $\widetilde{\nu}$ is the image of $\nu$ ${ }_{224}$ through the application $x \rightarrow \log (1+x)$ and $\widetilde{\gamma}=\gamma$. The solution of (4.10) is 225 therefore

$$
X_{t}=L_{\infty}\left(1-\mathrm{e}^{-\widetilde{Z}_{t+t_{0}}}\right) .
$$

226 It is easy to compute the mean $\mathrm{E}\left(X_{t}\right)$, as the quantity $\mathrm{E}\left(\mathrm{e}^{-\widetilde{Z}_{t+t_{0}}}\right)$ is the

Laplace transform at $\theta=1$ of the r.v. $Z_{t+t_{0}}$, which is easy to compute thanks to $(2.4)$ and (2.5):

$$
\mathrm{E}\left(X_{t}\right)=L_{\infty}\left(1-\mathrm{e}^{-\left(t+t_{0}\right) \widetilde{\phi}(1)}\right)
$$

Hence also for this model whatever the subordinator that is chosen (provided its Lévy measure vanishes outside ]0,1]), the mean of the solution of (4.10) follows a von Bertalanffy-type equation.

Note 4.1 Recall that the VBGF (1.1) is the solution of equation (4.10) when $Z$ is the deterministic subordinator $Z_{t}=k t$. Therefore (4.10) appears as a natural stochastic extension of the VBGF.

Remark also that some models already present in the literature are particular instances of (4.10). It is the case of the model (3.4) of Lv \& Pitchford (2007) where the authors actually consider $Z_{t}=r t+\sigma B_{t}$.

We point out again that the solution to (4.10) in this case does not have paths which are monotonically increasing. 
The solution (4.13) however has a drawback as a growth model because $X_{t} \rightarrow L_{\infty}$ as $t \rightarrow+\infty$ (unless $Z_{t} \equiv 0$ ). This implies that $\operatorname{Var}\left(X_{t}\right) \rightarrow 0$ as $t \rightarrow+\infty$, which is not realistic, as it would imply that all individuals should reach the same limiting size as $t$ increases. In order to overcome this problem it seems natural to assume that the extremal size $L_{\infty}$ is itself a random variable, thus accounting for the individual variability. In this way the two sources of randomness, $L_{\infty}$ and $\left(Z_{t}\right)_{t}$, appearing in equation (4.10) have the meaning of modeling the random individual variability and the environmental randomness respectively. It is therefore natural to assume that $L_{\infty}$ and $\left(Z_{t}\right)_{t}$ are independent. In this case formula (4.14) remains valid if one replaces $L_{\infty}$ by $\mathrm{E}\left(L_{\infty}\right)$. Therefore the expectation $\mathrm{E}\left(X_{t}\right)$ still follows a von Bertalanffy-type pattern as in (4.14), but with $L_{\infty}$ replaced by $\mathrm{E}\left(L_{\infty}\right)$. Similarly it is easy to compute the variance which is given by

$$
\begin{gathered}
\operatorname{Var}\left(X_{t}\right)=\operatorname{Var}\left(1-\mathrm{e}^{-\widetilde{Z}_{t+t_{0}}}\right) \mathrm{E}\left[L_{\infty}^{2}\right]+\operatorname{Var}\left(L_{\infty}^{2}\right) \mathrm{E}\left[1-\mathrm{e}^{\left.-\widetilde{Z}_{t+t_{0}}\right]^{2}}=\right. \\
=\left(\mathrm{e}^{-\left(t+t_{0}\right) \widetilde{\phi}(2)}-\mathrm{e}^{-2\left(t+t_{0}\right) \widetilde{\phi}(1)}\right) \mathrm{E}\left[L_{\infty}^{2}\right]+\operatorname{Var}\left(L_{\infty}^{2}\right)\left(1-\mathrm{e}^{-\left(t+t_{0}\right) \widetilde{\phi}(1)}\right)^{2} .
\end{gathered}
$$

Notice that $\operatorname{Var}\left(X_{t}\right)$ appears as the sum of two terms. As both $\widetilde{\phi}(1)$ and $\widetilde{\phi}(2)$ are positive, the first term vanishes at $-t_{0}$ and at $+\infty$ and it easy to see that (unless $Z_{t} \equiv 0$ ) it increases at first and then decreases. The second one conversely is increasing in $t$. Therefore the variance of $X_{t}$, according to different values of the parameters, can exhibit two possible behaviours:

- increasing or

- first increasing and then decreasing.

See Figure 2 for an illustration of this typical behaviour. 
The non monotonic behaviour of the variance is an interesting feature that is enjoyed also by some of the models proposed by Gudmundsson, 2005 and Lv \& Pitchford, 2007. This is not surprising as the computation above only makes use of the fact that $\left(X_{t}\right)_{t}$ is the solution of equation (4.10) with a driving process $\left(Z_{t}\right)_{t}$ which is a Lévy process, possibly a Brownian motion as it happens to be the case for the models of the authors above.

Note 4.2 In $\S 6$ we adapt the models of this section and of $\S 3$ to a population of herrings. It is fair however to point out a limitation that arises when trying to model real populations with processes driven by subordinators. Recall that the driving subordinator is meant to model the randomness of the growth process arising from the environment. The assumption of independence and stationarity of the increments of the subordinator implies the assumption that the environment remains stable and stationary in time, which is a feature that can be expected to hold in real world only for a short span of time, as the effect of season and of other sources of modification of the natural habitat should introduce a time-dependent effect. It is clear however that more realistic models would be far more complicated and, possibly, intractable in practice. The same observation, by the way, applies to the VBGF, which is an equation that is derived under the assumption of stationarity of the environment.

In the rest of this section we give more details in two particular cases, making assumptions on $Z$ that imply that $\widetilde{Z}$ is either a Gamma or an inverse Gaussian process. 
${ }_{284}$ Assume that the Lévy measure $\nu$ of $Z$ has a density $h$ with respect to the

Lebesgue measure. $h$ must be $\geq 0$, must vanish on $[1,+\infty[$ and be such that

$$
\int_{0}^{1} y h(y) d y<+\infty
$$

286

Then $-Z$ has a drift $=-\gamma$ and a Lévy measure $\bar{\nu}$ given by the density

$$
\bar{h}(y)=h(-y)
$$

287 The image of the measure $\bar{h}(y) d y$ by $y \rightarrow \log (1+y)$ is

$$
\widetilde{h}(y)=\mathrm{e}^{-y} h\left(1-\mathrm{e}^{-y}\right)
$$

Example 4.3 (The Gamma process) Let us assume that

$$
h(y)=\frac{\alpha}{-\log (1-y)}(1-y)^{\lambda-1}
$$

289 for $0<y \leq 1$ and $h(y)=0$ for $y>1$, where $\alpha>0, \lambda>0$. With this choice 290 we obtain

$$
\widetilde{h}(y)=\frac{\alpha}{y} \mathrm{e}^{-\lambda y}
$$

291 which is the Lévy measure of a Gamma process. Hence if we choose a driving 292 subordinator $Z$ with a Lévy measure as in (4.16) and drift $\gamma$, the solution of 293 (4.10) is

$$
X_{t}=L_{\infty}\left(1-\mathrm{e}^{-\gamma t-\widetilde{Z}_{t+t_{0}}}\right)
$$

294 where $\widetilde{Z}$ is a Gamma process with parameters $\alpha$ and $\lambda$ and drift $\gamma$. Thanks 295 to (4.10) and (2.7), for this model the mean is equal to

$$
\mathrm{E}\left(X_{t}\right)=\mathrm{E}\left(L_{\infty}\right)\left(1-\mathrm{e}^{-\gamma\left(t+t_{0}\right)}\left(\frac{\lambda}{\lambda+1}\right)^{\alpha\left(t+t_{0}\right)}\right)
$$


which, as already noted, is a von Bertalanffy equation with

$k=\gamma+\alpha \log \frac{\lambda+1}{\lambda}$. The variance of $X_{t}$ is also easily computed using (4.15):

$$
\begin{gathered}
\operatorname{Var}\left(X_{t}\right)=\mathrm{E}\left[L_{\infty}^{2}\right] \mathrm{e}^{-2 \gamma\left(t+t_{0}\right)}\left[\left(\frac{\lambda}{\lambda+2}\right)^{\alpha\left(t+t_{0}\right)}-\left(\frac{\lambda}{\lambda+1}\right)^{2 \alpha\left(t+t_{0}\right)}\right]+ \\
+\operatorname{Var}\left(L_{\infty}\right)\left[1-\mathrm{e}^{\gamma\left(t+t_{0}\right)}\left(\frac{\lambda}{\lambda+1}\right)^{\alpha\left(t+t_{0}\right)}\right]^{2}
\end{gathered}
$$

It is also possible to compute the density of $X_{t}$, conditioned to the value of $L_{\infty}=l$, which is given by

$$
f(x)=\frac{\lambda^{\alpha\left(t+t_{0}\right)}(l-x)^{\lambda-1}}{\Gamma\left(\alpha\left(t+t_{0}\right)\right) l^{\lambda}}\left(-\log \left(1-\frac{x}{l}\right)-\gamma\left(t+t_{0}\right)\right)^{\alpha\left(t+t_{0}\right)-1} \mathrm{e}^{\lambda \gamma\left(t+t_{0}\right)}
$$

for $l\left(1-\mathrm{e}^{-\gamma\left(t+t_{0}\right)}\right) \leq x \leq l$ and $f(x)=0$ otherwise. The graph of this density for different values of $t$ is produced in Figure 3. See in Figure 4 the graph of some simulated paths of $\left(X_{t}\right)_{t}$ with $\gamma=0$.

Example 4.4 (The inverse Gaussian process) Let us assume that $Z$ is a Levy process such that $\widetilde{Z}$ is an inverse Gaussian process with drift $\gamma$. If the Lévy measure of $Z$ has a density

$$
h(x)=\frac{1}{\sqrt{2 \pi \rho}(-\log (1-x))^{3 / 2}}(1-x)^{\frac{b^{2}}{2 \rho}-1}
$$

for $0<x<1$ and $h(x)=0$ elsewhere, then it turns out that $\widetilde{Z}$ is an inverse Gaussian process with parameters $b$ and $\rho$. The density of $X_{t}=L_{\infty}\left(1-\mathrm{e}^{-\widetilde{Z}_{t}-\gamma t}\right)$ given $L_{\infty}=l$ is straightforward to compute and is

$$
f_{t}(x)=\frac{t e^{b t / \rho}}{\sqrt{2 \pi \rho}}\left(-\log \left(1-\frac{x}{l}\right)\right)^{-3 / 2}\left(1-\frac{x}{l}\right)^{\frac{b^{2}}{2 \rho}-1} \exp \left(\frac{t^{2} \rho}{2 \log \left(1-\frac{x}{l}\right)}\right)
$$

for $0<x<l$ and $f_{t}(x)=0$ otherwise. 
One could also think of $\widetilde{Z}$ as a stable process with exponent $\alpha=\frac{1}{2}$. This choice however does not seem really suitable. Indeed in this case it is also possible to compute the density, as the same type of computation produces in this case the density, conditional on $L_{\infty}=l$,

$$
g_{t}(x)=\frac{t+t_{0}}{\sqrt{2 \pi}(l-x)\left(\log \frac{l}{l-x}\right)^{3 / 2}} \exp \left(-\frac{\left(t+t_{0}\right)^{2}}{2 \log \frac{l}{l-x}}\right), \quad 0<x<l .
$$

(with $\gamma=0$ ). The expression of this density suggests that the use of stable subordinators is inappropriate in order to model fish growth, as $g(x) \rightarrow+\infty$ as $x \rightarrow l-$, for every value of $t$.

The model based on the Doléans exponential (4.10) appears to enjoy many interesting features. It is fair however to point out that in order to take advantage of it there remains the question of determining an appropriate distribution for the maximal length $L_{\infty}$. It also appears difficult to find a distribution such that the density distribution of the length of the individuals of a given time $t, L_{\infty}\left(1-\mathrm{e}^{-Z_{t+t_{0}}}\right)$ has an explicit analytic expression. This density can be computed numerically, but it may be impossible to use of statistical methods based on maximum likelihood.

The question of determining a good candidate of distribution has been already tackled in (Sainsbury, 1980), where the author suggested a Gamma distribution.

\section{The Dataset}

The data used in this study were collected as part of the EU FP5 project "HERGEN" (Mariani et al., 2005; Ruzzante et al., 2006), and include a 
homogeneous collection of genetically and phenotypically characterized

North Sea Autumn Spawning herring (Clupea harengus L., 1758), from ICES

areas zones IVa, IVb and IVc. Herring is the dominant converter of

zooplankton production, using the biomass of copepods, mysids, euphausids

in the pelagic environment of the northern hemisphere (Winters \& Wheeler,

1987). Additionally, herring is a central prey item for higher trophic levels.

The spawning period of this species in the western North Sea is

September/October.

The samples were collected east of the Shetland Islands in July 2005. At that

moment, fish were located in that area to feed (summer feeding aggregation).

Aging was performed by counting the otolith (sagitta) winter rings, following standard procedures (Ruzzante et al., 2006; AA.VV, 2007). This method was

validated and tested for reliability following the procedure reported in

Beverton et al. (2004). The dataset is composed by 1255 specimens

belonging to 7 cohorts. The abundances and the ages of these cohorts are

showed in Table 1. The values of the empirical means and variances of the cohorts can be found in Fig 5.

\section{Analysis of the herring dataset}

In order to apply the models developed in $\S 3$ and $\S 4$ to the analysis of a

dataset as the herring one described in the previous section, one is

confronted with two kind of problems.

The first one is the determination of the appropriate driving subordinator.

Actually subordinators form a large family, every Lévy measure on $\mathbb{R}^{+}$

satisfying (2.6) being associated to a corresponding subordinator. This is 
therefore a non parametric problem and it appears very difficult to

determine this Lévy measure starting from data in the form of cohorts, as is the case for the herring dataset.

The second order of problems comes from the fact that these models are to be considered only approximatively correct, as they do not take into account the time variability of the environment (see Note 4.2).

In this section we produce the results obtained fitting the models of $\S 3$ and 4 to the herring dataset. In both cases we decided to work with the Gamma model, as it seemed to give better results than the inverse Gaussian.

In order to have a benchmark for the analysis of the dataset, we shall first adapt a normal Gaussian model inside each cohort. This is the default choice in literature (Imsland et al., 1998). Given the age $t_{i}$, the length $X_{t_{i}}$ is assumed to be normally distributed with mean $L_{\infty}\left(1-\mathrm{e}^{-k\left(t_{i}+t_{0}\right)}\right)$ and variance $\sigma_{i}^{2}$ (different cohorts are allowed to have different variances). This model requires therefore a number of parameters that is equal to $3+$ the number of cohorts.

It is worth noting that this is not a mixture model, as we are able to assign every individual to its class. Recall also that, as stated in the introduction, this is not a Individual Based Model.

The normal and time change models have been estimated by means of maximum likelihood, which appears to be the most natural method for them. As already pointed out in $\S 4$, for the Doléans exponential model, which is the most promising, the maximum likelihood method is inapplicable, as the distribution of the maximal size $L_{\infty}$ is not known. We therefore resorted to the least squares method in order to fit the moments. 
The estimates obtained with different models are shown in Table 2.

It is apparent that the normal model has a good agreement with the data, which is not surprising, given also the availability of many parameters.

\subsection{Time change model}

As already hinted in $\S 3$ this kind of models shows a certain rigidity. We give the estimates obtained using it for completeness sake only. Assuming that the lengths at time $t_{i}$ follow a $\Gamma\left(\alpha\left(1-\mathrm{e}^{-k\left(t_{i}+t 0\right)}\right), \lambda\right)$ distribution, we have a model with 4 parameters (whatever the number of cohorts). The estimated values are $\alpha=388.2, \lambda=1.33, k=0.64$ and $t_{0}=0.047$. From this we deduce $\hat{L}_{\infty}$ as the ratio $\hat{\alpha} / \hat{\lambda}$ (see Table 2 ). Estimates of the means and variances are obtained using (3.9).

Notice that the estimated means are very close to the empirical means (with the exception of cohort 1). However it is clear that this model cannot accommodate the variances. Recall that for this model the variances are necessarily increasing with time, at a difference with the behaviour of the empirical variances.

As already mentioned above, the model based on time change is a particularly parsimonious explanation of the data in terms of number of parameters, but has limited capacities of catching some relevant features. 


\subsection{Doléans exponential}

We tried a model based on the solution of (4.10) with respect to a Gamma process, as in Example 4.3.

According to this model, the density of the observations follows a distribution, given $L_{\infty}=l$, that is given in (4.20). Noting that the mean values of the lengths of the different cohorts must lie on the VBGF, we decided to perform a two-stage method of moments, in which, at first, the empirical means are interpolated by a VBGF function (1.1), therefore estimating the parameters $\mathrm{E}\left(L_{\infty}\right), k$ and $t_{0}$. In the second stage, we interpolated the empirical variances with the function (4.19). In this way we obtained estimates of $\alpha, \lambda, \gamma$ together with an estimate of $\sigma_{L_{\infty}}^{2}=\operatorname{Var}\left(L_{\infty}\right)$. Recall that the quantities $k$ and $\alpha, \lambda, \gamma$ are related by the constraint $k=\gamma+\alpha \log \frac{\lambda+1}{\lambda}$. For both stages we used a least squares method in order to fit the moments.

See Fig 5 for the graph of the fitted von Bertalanffy function and the empirical means with error bars.

This method produces an estimate of the mean and variance of $L_{\infty}$ without assuming any distribution for $L_{\infty}$. The estimates are $\alpha=1.3, \lambda=15$, $\gamma=0.55, k=0.63, t_{0}=0.072, \mathrm{E}\left(L_{\infty}\right)=297.3$ and $\sigma_{L_{\infty}}^{2}=160$. Finally, in order to obtain a concrete and visually appreciable estimate of the density of each cohort, we computed it numerically assuming for $L_{\infty}$ a Gamma distribution having parameters matching the estimated mean and variance, that is with parameters $\alpha_{0}=552.34$ and $\lambda_{0}=1.86$. Figure 6 produces a comparison between the density obtained as described above from the model 
and an empirical density, produced from the data with a usual kernel

estimator. Taking into account the limited number of parameters employed

by this model one can appreciate the nice fit for some of the cohorts.

Discrepancies can be found for the first two cohorts and for the sixth one.

For the fifth one there is a evident discrepancy: notice however that, as

pointed out in Fig 5, also the estimated VBFG curve that fits the mean of this fifth cohort. At this point one should keep in mind Note 4.2.

\section{Discussion}

Although several stochastic model are available in the literature in order to compute the time evolution of the distribution of the size of a population, a certain number of drawbacks still remain, leading to the need of a more appropriate formulation that is able to take into account some key aspects of animal growth.

These aspects basically concern the inclusion of the different sources of variability in growth rate among individuals, which directly determine the size density observed at different ages, and the property of the growth process of being increasing. In this work we developed two models based on the use of subordinators as driving processes. Our aim was to provide a new stochastic model, mainly of theoretical interest for the description of the growth process for a large class of organisms. It turns out that one of them, the model based on the Doléans exponential giving rise to a Gamma process, is also able to produce an appreciable fit with the data. In particular it is able to reproduce the non monotonic trend of the variance, that is the measure of intra-cohort variability of size. This is an important feature: a 
reduction of the demographic variance starting at some time is often observed in data (Gudmundsson, 2005 e.g.).

In general, the variability in individual size increases within the same cohort through time when the individual growth rate is positively correlated with itself in time (Gudmundsson, 2005). This phenomenon is called "growth depensation".

Conversely, a reduction of the cohort variance (the so called "growth compensation") has been up to now explained with the fact that survival of individuals are not independent and identically distributed (Kendall \& Fox, 2002). In other words, there needs to be some systematic structure in the population. Common biological mechanisms that can produce a reduction of demographic variance are contest competition (including territoriality), long-lived individuals with lifetime demographic traits ("individual heterogeneity"), maternally imposed variation, and directional or balancing selection.

Observe however that our model explains this typical behaviour of the variance without introducing an explicit selection mechanism related to some size-dependent mortality. By this we do not claim that such a mechanism does not exist (and we think that it might be natural to devise a more sofisticated model including this feature), but simply that it is not necessary in order to explain the observed behavior of the empirical variances.

The possibility to model and predict this aspect of fish growth seems to be of great importance in both theoretical studies and fishery management applications. This is particularly true if referred to the increasing use of measurement of the growth pattern of organisms (like fish) as an ecological 
indicator (Bennett et al., 1995). Further, assessment of growth pattern, combined with other measurement of physiological condition, have the potential to yield information on the history of environmental stress (e.g. from contaminants) or selective pressures (e.g. fisheries) and adaptation to environmental change (Jorgensen et al., 2007). In fact, statistical analyses of long-term data from some exploited fish stocks have revealed evolutionary changes in reaction norms (Ernande et al., 2004). Accuracy of the growth model at the basis of these analyses obviously affect the results, so that the development of sound approach to model and predict size distribution is a main target.

A future direction will concern the assessment of the effect of the aging error on the estimates obtained using our model. Even if the aging method is reliable, it seems that errors in aging young cohorts can significantly affect the estimates. This is due to the fact that both VBGF and the variance function converge with age.

The present work represent a first attempt to apply the Lévy processes to the subject. We think that these and the ideas developed in this paper can be adapted to introduce stochasticity into more complex models of growth (see Edwards et al., 2007).

\section{Conclusion}

The aim of the present work was the development of a stochastic model of growth, and therefore an Individual Based Model for the distribution of data, that is compatible with the von Bertalanffy function and might be able to catch more of the features empirically observed. 
The model developed in relation with the Doléans exponential shows interesting features. As other models already in the literature (Wang, 1999;

Gudmundsson, 2005; Lv \& Pitchford, 2007) it is able to explain the observed non monotonic behaviour of the variances. However, it is the first stochastic model producing paths that are increasing, thus, giving a realistic random model of individual growth.

Our main objective was mainly theoretical, but we think that the models developed in $\S 4$ can be of interest for practical applications such as stock assessment and forecasting. In this direction however some questions require a deeper investigation.

These are

- The determination (elicitation) of a suitable distribution for the maximal size $L_{\infty}$

- The determination of the appropriate subordinator.

\section{References}

AA.VV (2007). Report of Herring Assessment Working Group for the Area south of 628 N. ICES CM 2007/ACFM: 11.

Arino, O., Shin, Y. \& Mullon, C. (2004). A mathematical derivation of size spectra in fish populations. C.R. Biol. 327, 245-254.

Bennett, W., Ostrach, D. \& Hinton, D. (1995). Condition of larval striped bass in a graught-stricken estuary: evaluating pelagic food web limitation. Ecol. Appl. 5, 680-692.

Beverton, R., Hylen, A., Østvedt, O.-J., Alvsvaag, J. \& Iles, T. 
(2004). Growth, maturation, and longevity of maturation cohorts of Norwegian spring-spawning herring. ICES J.Mar.Scie. 61, 165-175.

Brett, J. (1979). Environmental factors and growth. In: Fish Physiology, Vol. III, Bioenergetics and Growth. New York: Academic Press Inc.

Caswell, H. (2001). Matrix population models: construction, analysis, and interpretation. Sunderland, Massachusetts, USA: Sinauder Associated.

Conover, D. \& Munch, S. (2002). Sustaining fisheries yields over evolutionary time scales. Science 297(5578), 94-96.

Cont, R. \& Tankov, P. (2004). Financial modelling with jump processes. Chapman \& Hall/CRC Financial Mathematics Series. Chapman \& Hall/CRC, Boca Raton, FL.

DeAngelis, D., Rose, K., Crowder, L., Marshall, E. \& Lika, D. (1993). Fish cohort dynamics: Application of complementary modelling approaches. Am. Nat. 142(4), 604-622.

Edwards, A., Phillips, R., Watkins, N., Freeman, M., Murphy, E., Afanasyev, V., Buldyrev, S., Da Luz, M., Raposo, E. \& Stanley, H. (2007). Revisiting Lévy flight search patterns of wandering albatrosses, bumblebees and deer. Nat. 449, 1044-1049.

Ernande, B., Dieckmann, U. \& Heino, M. (2004). Adaptive changes in harvested populations: plasticity and evolution of age and size at maturation. Proc. R. Soc. Lond. 271(1537), 415-423.

Essington, T., Kitchell, J. \& Walters, C. (2001). The von Bertalanffy growth function, bioenergetics, and the consumption rates of fish. Can. J. Fish. Aquat. Sci. 58, 2129-2138.

Fujiwara, M., Kendall, B. \& Nisbet, R. (2004). Growth autocorrelation and animal size variation. Ecol. Lett. 7, 106-113.

Gudmundsson, G. (2005). Stochastic growth. Can. J. Fish. Aquat. Sci. 
62, 1746-1755.

Hinrichsen, H.-H., Buehler, V. \& Clemmesen, C. (2007). An

individual-based model for direct conversion of otolith into somatic growth rates. Fish.Oceanogr. 16(3), 207-215.

Imsland, A., Jonassen, T., Langston, A., Hoare, R., Wergeland, H., FitzGerald, R., Mulcahy, M. \& Stefansson, S. (2002). The interrelation of growth performance and disease resistance of juvenile atlantic halibut (hippoglossus hippoglossus l.) from different populations. Aquacul. 204, 167-177.

Imsland, A., Nilsen, T. \& Folkvord, A. (1998). Stochastic simulation of size variation in turbot: possible causes analysed with an individual based-model. J. Fish. Biol. 53, 237-258.

Jorgensen, C., Enberg, K., Dunlop, E., Arlinghaus, R., Boukal, S., Brander, K., Ernande, K., Gardmark, A., Johnston, F., Matsumura, S., Pardoe, H., RaAb, K., Silva, A., Vainikka, A., Dieckmann, U., Heino, M. \& RijnsdorP, A. (2007). Managing evolving fish stocks. Science 318, 1247-1248.

Karlin, S. \& TAYlor, H. M. (1981). A second course in stochastic processes. New York: Academic Press Inc. [Harcourt Brace Jovanovich Publishers].

Kendall, B. \& Fox, G. (2002). Variation among individuals and reduced demographic stochasticity. Cons. Biol. 16(1), 109-116.

Kooluman, S. (2000). Dinamic energy and mass budgets in biological systems. Second edition. Cambridge, UK: Cambridge University Press Inc.

Lv, Q. \& Pitchford, J. W. (2007). Stochastic von Bertalanffy models, with applications to fish recruitment. J. Theoret. Biol. 244(4), 640-655.

Mariani, S., Hutchinson, W., Hatfield, D., E.M.C. Ruzzante, 
Simmonds, T. A. C., E.J. Dahlgren, Brigham, J., Torstensen, E. \& Carvalho, G. (2005). Biocomplexity in a highly migratory marine pelagic fish, atlantic herring. Mar. Ecol. Progr. Ser. 303, 245-257.

Mulligan, T. \& Leaman, B. (1992). Length-at-age analysis: Can you get what you see? Can. J. Fish. Aquat. Sci. 49, 632-643.

Rilling, G. \& Houde, E. (1999). Regional and temporal variability in the distribution and abundance of bay anchovy (anchoa mitchilli) eggs, larvae, and adult biomass in the chesapeake bay. Estuar. 22, 1096-1109.

Ruzzante, D., Mariani, S., Bekkevold, D., Andre, C., Mosegaard, H., Clausen, L., Dahlgren, T., Hutchinson, W., Hatfield, E., Torstensen, E., Brigham, J., Simmonds, E., Laikre, L., Larsson, L., Stet, R., Ryman, N. \& Carvalho, G. (2006). Biocomplexity in a highly migratory marine pelagic fish, atlantic herring. Proc. R. Soc. Lond. 273, 1459-1464.

SAINsBURY, K. (1980). Effect of individual variability on the von Bertalanffy growth equation. Can. J. Fish. Aquat. Sci. 37, 241-247.

SATO, K.-I. (1999). Lévy processes and infinitely divisible distributions, vol. 68 of Cambridge Studies in Advanced Mathematics. Cambridge: Cambridge University Press. Translated from the 1990 Japanese original, Revised by the author.

Sumpter, J. (1992). Control of growth of rainbow trout (oncorhynchus mykiss). Aquacul. 100, 299-320.

UChMANSKI, J. (2000). Individual variability and population regulation: An individual-based model. Oikos 9, 539-548.

VON Bertalanffy, L. (1938). A quantitative theory of organic growth.

Human Biol. 10, 181-213.

vOn BertalanfFy, L. (1957). Quantitative laws in metabolism and 
growth. Quart. Rev. Biol. 32(3), 217-231.

WANG, Y. (1999). Estimating equations for parameters in stochastic growth models from tag-recapture data. Biometrics 55, 900-903.

Wang, Y. \& Thomas, M. (2003). Accounting for individual variability in the von Bertalanffy growth model. Can. J. Fish. Aquat. Sci. 52, $1368-1375$.

Weatherley, A. \& Gill, H. (1987). The biology of fish growth. New York: Academic Press Inc. [Harcourt Brace Jovanovich Publishers].

Winters, G. \& Wheeler, J. (1987). Clupeoid fishes of the world (suborder clupeioidei). an annotated and illustrated catalogue of the herrings, sardines, pilchards, sprats, shads, anchovies and wolf-herrings. Can. J. Fish. Aquat. Sci. 44, 882-900. 
Fig. 1

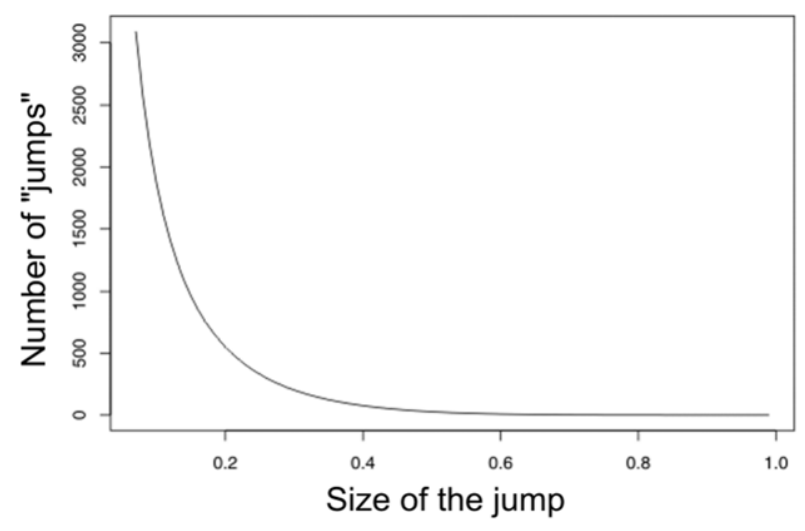


Fig. 2

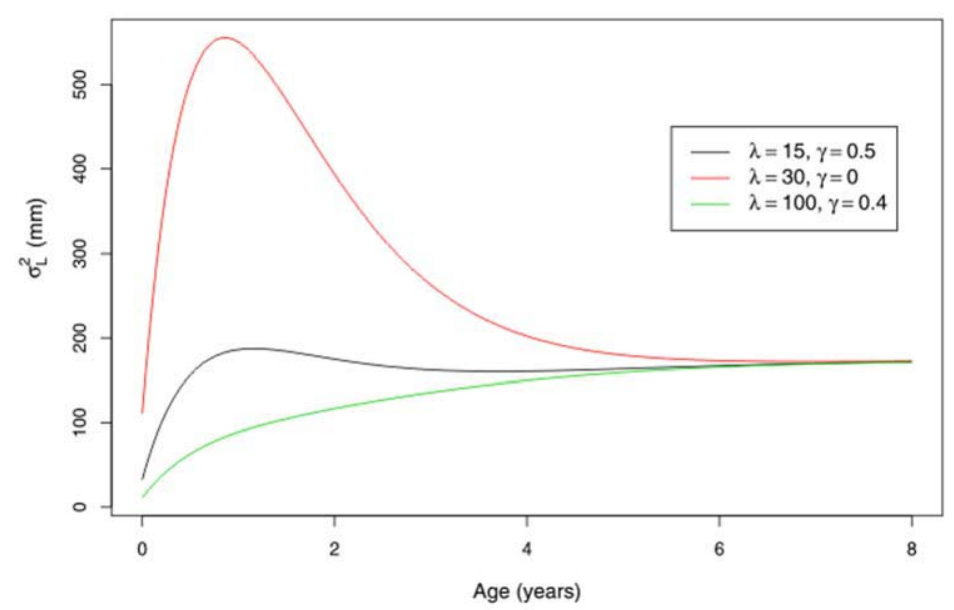


Fig. 3

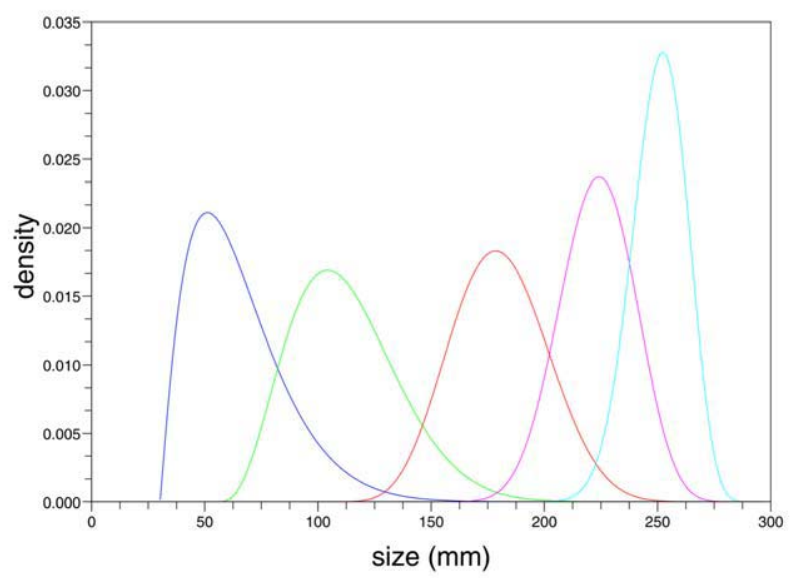


Fig. 4

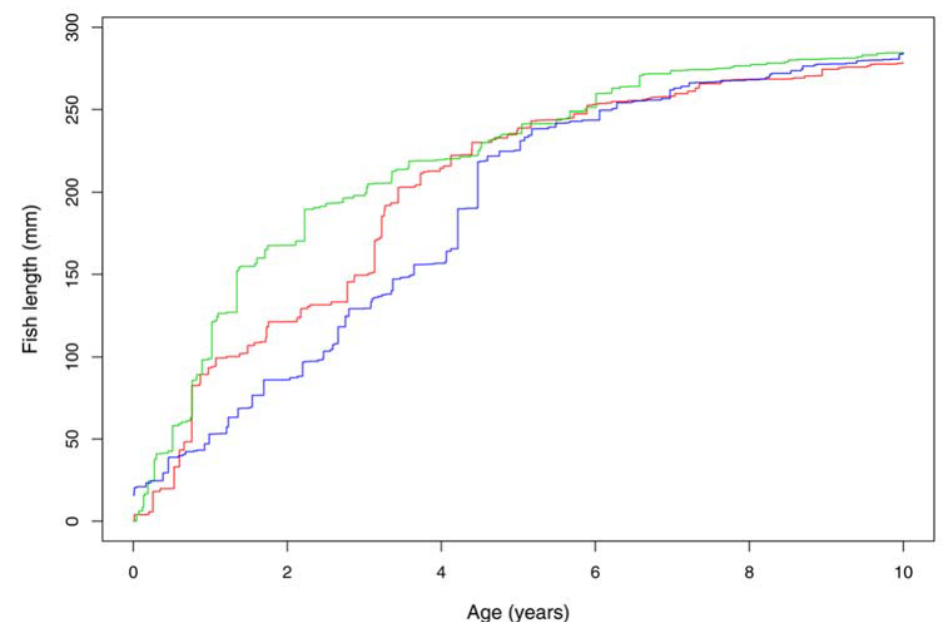


Fig. 5
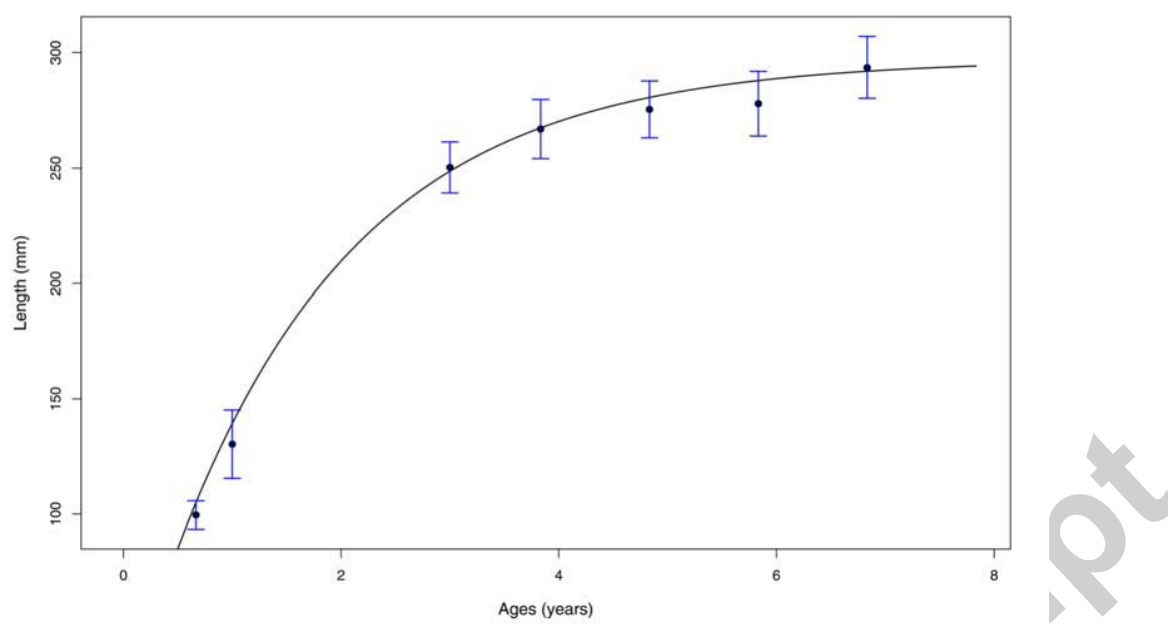
Fig. 6
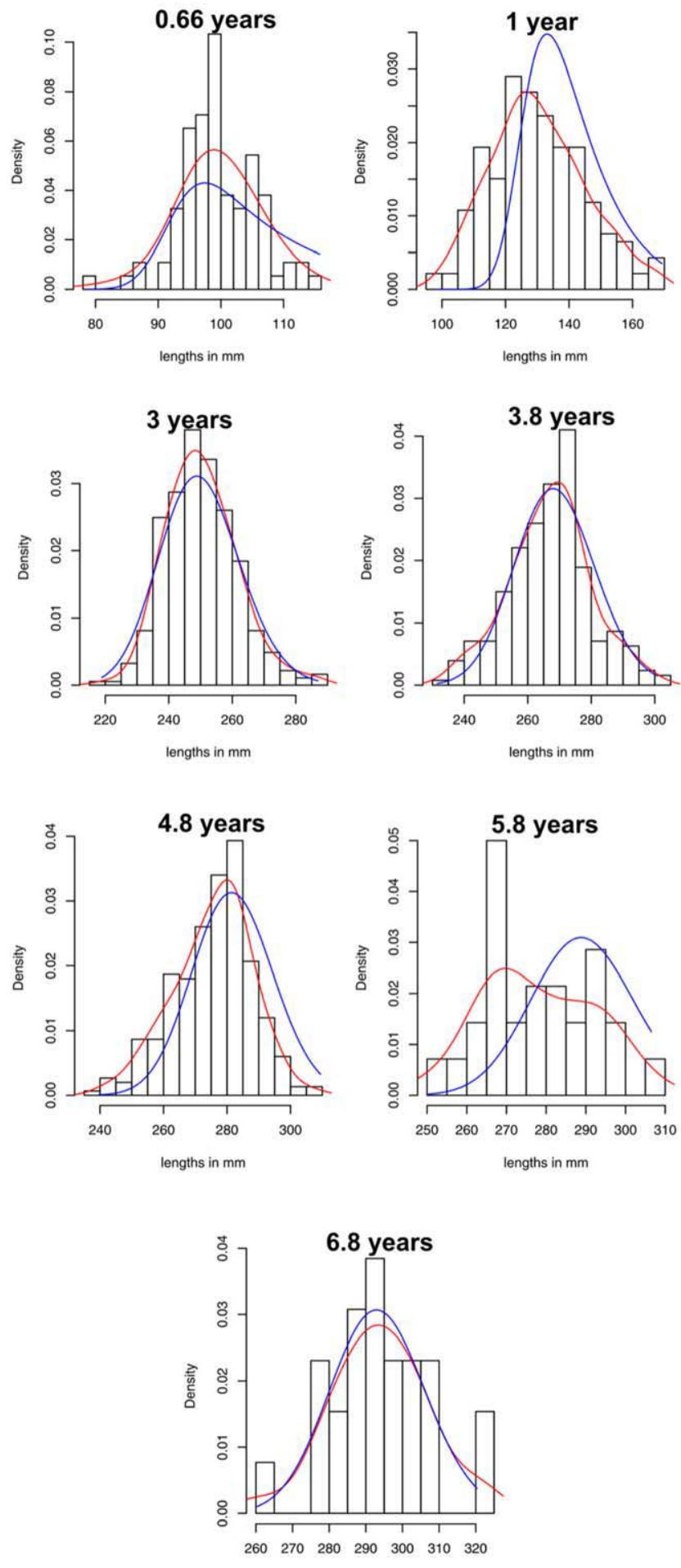

lengths in $\mathrm{mm}$ 